\title{
Health-related quality of life in patients with anterior cruciate ligament insufficiency undergoing arthroscopic reconstruction: a practice-based Italian normative group in comorbid-free patients
}

\author{
V. Calvisi $\cdot$ B. De Vincentiis $\cdot$ P. Palumbo · \\ R. Padua $\cdot$ S. Lupparelli
}

Received: 10 September 2008/Accepted: 5 October 2008/Published online: 29 October 2008

(C) Springer-Verlag 2008

\begin{abstract}
Background Health-related quality of life (HRQoL) in anterior cruciate ligament (ACL) insufficiency has not been assessed in comorbid-free patients to date. An observational study was therefore conducted on a practice-based sample to test the hypothesis that SF-36 scoring in patients with chronic ACL insufficiency differs from the age- and gender-matched Italian norm.

Materials and methods Chronically ACL-insufficient patients with or without meniscal and/or focal chondral lesions were enrolled in the study. Exclusion criteria were acute ACL tear, severe and diffuse chondral lesions, concomitant knee major ligamentous injuries and/or fractures requiring surgery, previous ACL surgery and infectious, neoplastic and inflammatory disease. Knee function was evaluated by International Knee Documentation Committee (IKDC) form, HRQoL with the SF-36 questionnaire, and associated medical comorbidities by a Self-Administered Comorbidity Questionnaire (SCQ).
\end{abstract}

\footnotetext{
V. Calvisi $(\bowtie) \cdot$ B. De Vincentiis · P. Palumbo · S. Lupparelli

Department of Orthopaedic Surgery, University of L'Aquila, Viale S. Salvatore, Edificio Delta 6, 67010 Coppito (AQ), L'Aquila, Italy

e-mail: calvisiv@libero.it

R. Padua

San Giacomo Hospital, Rome, Italy

R. Padua $\cdot$ S. Lupparelli

GLOBE (Italian Working Group for Evidence-Based

Orthopaedics), Rome, Italy

R. Padua

Fondazione Don Gnocchi, Rome, Italy
}

Results A total of 316 consecutive patients, 265 males and 51 females (median age 25 years, range 15-52 years) met the inclusion/exclusion criteria. SF-36 norm-based scoring showed that the Physical Functioning, Role Physical, Bodily Pain, and Social Functioning domains were significantly lower than the Italian norm; the Role Emotional domain was also lower than the norm, but the difference was not significant. Conversely, the General Health and Mental Health domains scored significantly higher than the norm; the Vitality domain also exceeded, albeit not significantly, the norm.

Conclusions The decision-making process leading to ACL reconstruction currently emphasises the evaluation of knee function and patients' level of activity. The findings in our study, by showing that chronic ACL insufficiency significantly affects HRQoL in otherwise healthy patients, suggest that a multidimensional evaluation including HRQoL in addition to knee function might be integrated into outcome assessment.

Keywords Anterior cruciate ligament - Reconstruction · Normative group $\cdot$ SF-36 $\cdot$ Quality of life

\section{Introduction}

Although the natural history of anterior cruciate ligament (ACL) insufficiency is unclear [1,2], it is generally agreed that recurrent episodes of symptomatic instability would lead to an accrual of joint damage [1,3], particularly if meniscectomy has been performed [4]. Current indications for ACL reconstruction include the desire of recreational or professional athletes for a prompt return to preinjury activity level or the persistence of symptomatic knee instability despite an appropriate rehabilitation program and activity level modification $[1,3]$. 
A number of studies suggested that ACL insufficiency would affect health-related quality of life (HRQoL) as assessed by the Medical Outcome Study 36-Item ShortForm Health Survey (SF-36) [5, 6]. It has also been advocated that assessment of HRQoL should be incorporated for outcome assessment in patients with ACL insufficiency [7]. However, a recent report demonstrated that comorbidity should be controlled when assessing HRQoL in candidates for ACL reconstruction because it acts as a confounder [8].

We have therefore assessed HRQoL using the SF-36 in comorbid-free recreational athletes with chronic ACL insufficiency undergoing arthroscopic reconstruction with the purpose of setting a normative HRQoL profile from a single surgeon's practice to be compared with the age- and gender-matched Italian norm. This may prove useful in clarifying the true impact of ACL insufficiency on HRQoL of candidates for surgery, at the same time yielding normative data that could be used in future studies to investigate whether SF-36 profiles also vary across practices.

\section{Materials and methods}

A prospective study was conducted on patients with ACL insufficiency who had received indication for arthroscopic reconstruction by the senior author (V.C.) and were admitted to our department from December 2003 to January 2008. Patients were included in the study when they had unilateral chronic ACL insufficiency with or without associated meniscal and/or focal chondral lesions and a normal function of the contralateral lower limb. We adhered to published criteria that regard an ACL tear as chronic 6 weeks after injury [9]. Patients with acute ACL tear, severe (Outerbridge grade III or IV) and diffuse chondral lesions, knee major (grade III) peripheral ligamentous injuries and/or fractures requiring surgery, previous ACL reconstructive surgery, and associated medical comorbidity were excluded.

The indication for ACL reconstruction was the persistence of a symptomatic knee instability after a 6-month rehabilitation programme in patients who cannot or will not change their activity level. Persistence of knee giving way despite rehabilitation and activity level modification was also an indication. The preoperative clinical diagnosis of ACL tear was based on history and clinical examination (positive Lachman and pivot-shift tests). Patients unable to relax their musculature during physical examination were evaluated under anaesthesia. Diagnosis also included assessment of tibial anterior translation (TAT) with KT-1000 knee ligament arthrometer (MEDmetric, San Diego, CA) at $67 \mathrm{~N}$ and $89 \mathrm{~N}$, and at maximal manual traction (MMT).
TAT exceeding $10 \mathrm{~mm}$ or a side-to-side difference (SSD) $>3 \mathrm{~mm}$ at MMT were considered indicative of ACL tear [10]. A diagnostic arthroscopy was performed on the day of index surgery before autograft harvesting. Following confirmation of the ACL injury, the presence of meniscal tears and/or chondral lesions was assessed.

Patients were requested to fill in the Italian validated version of the SF-36 [11] and a questionnaire assessing comorbidity, on the day before surgery. Administering SF-36 in proximity to surgery does not affect the outcome [12]. The SF-36 is a patient-based questionnaire including eight domains: Physical Functioning (PF), Role Physical (role limitations due to physical health problems) (RP), Bodily Pain (BP), General Health (GH), Vitality (VT), Social Functioning (SF), Role Emotional (role limitations due to emotional health problems) (RE), and Mental Health (MH) [13]. Scores of the SF-36 domains were computed according to validated calculation algorithms [14]. The Comorbidity Index (CI) used in the present study is obtained from a Self-Administered Comorbidity Questionnaire (SCQ) that has been validated for use in clinical research [15]. The SCQ lists 13 medical conditions (closed-ended items: heart disease; high blood pressure; lung disease; diabetes; ulcer or stomach disease; kidney disease; liver disease; anaemia or other blood disease; cancer; depression; osteoarthritis, degenerative arthritis; back pain; rheumatoid arthritis) plus three medical diseases (open-ended items) that may be specified by the patient. Patients are asked to indicate: (1) whether they have been diagnosed by a physician as affected by one or more of the listed medical conditions, (2) whether they receive treatment for that condition, and (3) whether patients' function is limited by that condition. A binary answer (yes or no) is supplied for each question. One overall CI score and three additional subscale scores (comorbidity subscale: 1 , have problems; 2 , receive treatment; 3 , limit activity) can be calculated. The overall CI was used for the present analysis. Knee function was assessed using the International Knee Documentation Committee (IKDC) examination form [16]. Norm-based scoring, which controls for age and gender as confounders, was used to calculate the differential SF-36 scores from the age- and gender-matched Italian norm for each patient [17-19].

Statistical analysis

Nonparametric tests (Mann-Whitney $U$ test and contingency tables) were used for comparison of categorical variables and of continuous variables for which no assumption of normality distribution was made. The mean difference from the age- and gender-matched norm (normbased scoring) and the relevant $95 \%$ confidence limits (CL) were computed for the SF-36 domains. The mean and standard deviation (SD) of the domain scores were also 
calculated to allow readers to perform $t$-test comparison using summary data. Although SF-36 scores are not continuous variables, the method has been previously reported in peer-reviewed literature [20]. The two-sided significance level for statistical inference tests was preset at $P<0.05$. Statistical analysis was performed using a dedicated software (StatsDirect version 2.5.7, StatsDirect Ltd, Cheshire, UK).

\section{Results}

A total of 316 consecutive patients, 265 males and 51 females (median age 25 years, range $15-52$ years) met the inclusion/exclusion criteria and constitute the study sample. The variables related to the study sample are reported in Table 1.

SF-36 norm-based scoring showed that the PF, RP, BP, and SF domains were significantly lower than the Italian norm. The RE domain was also lower than the norm but the difference was not significant. Conversely, the GH and $\mathrm{MH}$ domains were significantly higher than the norm. The VT domain score exceeded, albeit not significantly, the norm. The norm-based SF-36 profile along with the 95\% CLs relevant to each domain are illustrated in Fig. 1.

\section{Discussion}

The examination of patients with ACL insufficiency generally focuses on the assessment of knee function by physical and instrumented laxity testing. Clinical history is also important to the treating physician because it allows him/her to become aware of patient lifestyle and willingness to modify activity level. Hence, the indication for ACL reconstruction most frequently draws on the integration of patient- and physician-based assessment of knee disability [1, 3]. However, previous studies have demonstrated that disability related to musculoskeletal impairment also affects patient perception of HRQoL [21-23]; for example, comparison of SF-36 profiles among samples of patients with spine disorders has suggested that HRQoL modification may be disease related and that SF-36 profiles are capable of discriminating patients requiring intervention [24]. Thus, incorporation of SF-36 profiles into decision-making has been recommended in clinical practice $[25,26]$.

This is the first study investigating HRQoL modifications in a large and well-selected sample of chronically ACL-insufficient patients who have been recommended for surgery, the indications to surgery being based on explicitly stated criteria. The study sample only included patient with no associated medical comorbidities, which strengthens the
Table 1 Variables of patients with chronic anterior cruciate ligament insufficiency with indication for reconstruction

\begin{tabular}{|c|c|}
\hline Characteristics $(n=316)$ & \\
\hline Age (years) & \\
\hline Median & 25 \\
\hline Range & $15-52$ \\
\hline Gender & \\
\hline Males & $265 / 316(84 \%)$ \\
\hline Females & $51 / 316(16 \%)$ \\
\hline Knee side & \\
\hline Right & $168 / 316(53 \%)$ \\
\hline Left & $148 / 316(47 \%)$ \\
\hline IKDC classification & \\
\hline B & $154 / 316(49 \%)$ \\
\hline $\mathrm{C}$ & $149 / 316(47 \%)$ \\
\hline $\mathrm{D}$ & $13 / 316(4 \%)$ \\
\hline TAT $(\mathrm{mm}$ at MMT) & \\
\hline Mean \pm standard deviation & $10 \pm 3$ \\
\hline $\mathrm{SSD}(\mathrm{mm}$ at MMT) & \\
\hline Mean \pm SD & $5 \pm 3$ \\
\hline Isolated meniscal tears & $130 / 316(41 \%)$ \\
\hline Isolated chondral lesions $^{\mathrm{a}}$ & $9 / 316(3 \%)$ \\
\hline Associated meniscal and chondral lesions & $32 / 316(10 \%)$ \\
\hline Comorbidity index & \\
\hline Median & 0 \\
\hline Range & $0-0$ \\
\hline SF-36 domain scores (mean \pm standard de & \\
\hline $\mathrm{PF}$ & $85.18 \pm 13.51$ \\
\hline $\mathrm{RP}$ & $60.08 \pm 38.38$ \\
\hline $\mathrm{BP}$ & $77.62 \pm 21.78$ \\
\hline $\mathrm{GH}$ & $80.67 \pm 12.12$ \\
\hline VT & $73.60 \pm 46.72$ \\
\hline SF & $78.94 \pm 20.77$ \\
\hline $\mathrm{RE}$ & $81.16 \pm 31.59$ \\
\hline MH & $77.05 \pm 15.00$ \\
\hline
\end{tabular}

IKDC International Knee Documentation Committee, TAT tibial anterior translation, $M M T$ maximum manual traction, $S S D$ side-toside difference, $P F$ Physical Function, $R P$ Role Playing, $B P$ Bodily Pain, $G H$ General Health, $V T$ Vitality, $S F$ Social Function, $R E$ Role Emotional, $M H$ Mental Health

a Degree of chondral lesions according to Outerbridge classification

capability of SF-36 to reflect the true impact of ACL insufficiency on health status at the same time controlling for age and gender as additional confounders using normbased scoring. The results of our study confirmed the hypothesis that chronic ACL insufficiency significantly affects HRQoL compared with the norm. On the other hand the finding that the $\mathrm{GH}$ and $\mathrm{MH}$ domains scored significantly higher than the norm was somewhat surprising. Although any explanation is purely conjectural, it should be remembered that, unlike all other SF-36 scales, defining 


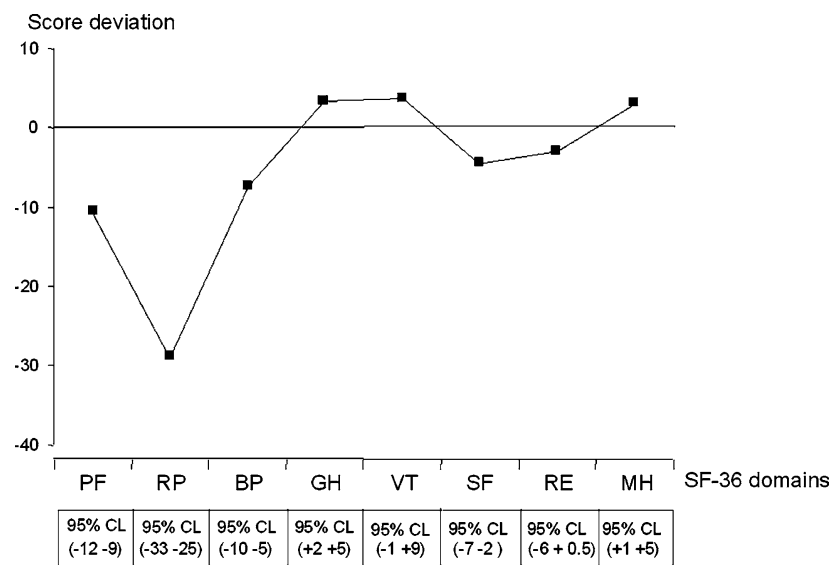

Fig. 1 Diagram showing the mean differences of the SF-36 domain scores in the study sample $(n=316)$ of candidates for anterior cruciate ligament reconstruction from the age- and gender-matched Italian norm. The abscissa line intersecting the ordinate line at the zero value represents the norm and SF-36 domains scoring above or below the reference line, respectively, indicate scores that are higher or lower than the norm. The confidence limits not including zero reported in the included table convey statistical significance. Domain legends: $P F$ Physical Functioning, $R P$ Role Physical (role limitations due to physical health problems), $B P$ Bodily Pain, $G H$ General Health, $V T$ Vitality, $S F$ Social Functioning, $R E$ Role Emotional (role limitations due to emotional health problems), $M H$ Mental Health

a positive health status as absence of limitation or disability and in which the maximum score is reached when no limitation or disability are reported, the $\mathrm{GH}$ and $\mathrm{MH}$ domains, as well as the VT domain, measure a wider range of positive and negative health states. In these scales an intermediate score is reached when individuals report no limitation or disability while the maximum score is achieved when patients enjoy a positive health status [27]. Thus it could be assumed that being on the waiting list for ACL reconstruction may cause a positive outcome bias in young patients willing to return to sports. The enhanced perception of well-being could be detected by those scales least affected by a ceiling effect. Whether higher scores in the GH and $\mathrm{MH}$ domains represent a characteristic pattern in patients with a chronic ACL injury who have been selected for surgery or a random variation effect observed in our sample remains a hypothesis to be tested.

To the best of our knowledge only two studies published in the peer-reviewed literature have focused on HRQoL modifications as measured by the SF-36 in ACL-insufficient patients $[5,6]$. In a prospective observational study Shapiro et al. [6] reported on 113 patients (65 males and 48 females with mean age 30 years, range $15-52$ years) with chronic ACL insufficiency. The injury was defined as chronic if lasting longer than 4 months since initial injury. Indication for ACL reconstruction was based on functional status assessment, including symptomatic instability for chronic injuries, pivot shift testing, TAT at MMT $>3 \mathrm{~mm}$ at KT-1000, activity levels, and willingness to modify activity levels. The sample was subdivided into four subgroups, namely 58 patients with an indication for surgery with surgery performed, 13 patients advised to undergo surgery without surgery performed, 37 patients not recommended for surgery undergoing conservative treatment, and 5 patients not recommended for surgery undergoing conservative treatment and subsequently operated on for chronic symptoms. The study analyzed the SF-36 variations at 6, 12, and 24 months follow-up as well as SF-36 correlation with the Lysholm scale, Tegner activity scale, and IKDC evaluation score. The authors found that the SF36 could not discriminate patients requiring surgery, that it showed significant treatment-related changes over time, and that the SF-36 scales correlated with both the Lysholm scale and the IKDC evaluation score. The study conclusion recommended that the SF-36 should be incorporated in the examination tools used for assessing the outcome in ACL-insufficient patients to enhance the value of treatment to policy makers. A retrospective cohort study by McAllister et al. [5] reported on 33 Division I-A athletes who had sustained an ACL injury 2-14 years before. They were matched to 33 control peers playing a similar role in a similar position. Twenty-nine of the 33 athletes had previously undergone ACL reconstruction. Patients were stratified into four classes according to the subjective portion of the IKDC evaluation score. The Tegner activity scale, the subjective portion of the IKDC evaluation score and the SF-36 domain scores relevant to the study and control groups as well as to the ACL-reconstructed and ACL-unreconstructed subgroups were reported. The authors concluded that quality of life in ACL-injured elite collegiate athletes did not differ from that of uninjured peers although knee function and the number of athletes competing at an elite level varied among groups. One limitation of these studies is that SF-36 scores did not control for comorbidity. Also, a limited number of baseline variables were used to characterize the study sample and the indications for surgery were not explicitly stated.

Considering that McAllister et al. found that SF-36 profiles in healthy and injured elite collegiate athletes differed from the norm [20], we suggest that normative data derived from large samples of patients with ACL insufficiency belonging to different practices should be obtained prior to comparing SF-36 profiles. A paper by Harryman et al. recently demonstrated that patients with rotator cuff tears from different practices differed in their general health, among a set of other variables [28]. The authors concluded that samples collected from multiple practices cannot be assumed to stem from the same population. This is an important issue when appraising the literature as well as when collecting data for national registries, as we cannot rule out the hypothesis that 
surgeon-related and sociocultural variables are confounders, as well as age, gender, and comorbidity, affecting SF-36 scoring.

Two limitations of our study could not be avoided. One is that the sample composition included predominantly males. Marx et al. [3] reported that 5.4\% of 261 surgeons of the American Academy of Orthopaedic Surgeons (AAOS) regard female sex as a negative factor influencing their indication for surgery. The senior surgeon of our study (V.C.) does not consider female sex as a negative predictor for surgery. Alternatively, the gender imbalance could be related to unequal participation of Italian males and females in sports [29], however this confounder was controlled for by norm-based scoring. Secondly, the lack of a control group of patients with ACL insufficiency not undergoing reconstructive surgery did not allow to assess whether indication for surgery is an additional confounder affecting SF-36 profiles and whether the SF-36 would be capable of discriminating patients requiring surgery.

In conclusion, the decision-making process leading to ACL reconstruction currently emphasizes the evaluation of knee function and patient level of activity. The findings in our study are in agreement with published papers suggesting that a multidimensional evaluation including HRQoL in addition to knee function might be integrated into preoperative patient evaluation. Finally, future research may help clarify whether candidates for ACL reconstruction share distinctive patterns of HRQoL modifications and whether SF-36 profiles also vary in relation to the source practice.

Conflict of interest statement No funds were or will be received in support of this work. No benefits in any form have been or will be received from a commercial party related directly or indirectly to the subject of this manuscript.

\section{References}

1. Beynnon BD, Johnson RJ, Abate JA, Fleming BC, Nichols CE (2005) Treatment of anterior cruciate ligament injuries, part I. Am J Sports Med 53:1579-1602

2. Linko E, Harilainen A, Malmivaara A, Seitsalo S (2005) Surgical versus conservative interventions for anterior cruciate ligament ruptures in adults. Cochrane Database Syst Rev (2):CD001356

3. Marx RG, Jones EC, Angel M, Wickiewicz TL, Warren RF (2003) Beliefs and attitudes of the American Academy of Orthopaedic Surgeons regarding the treatment of anterior cruciate ligament injury. Arthroscopy 19:762-770

4. Maffulli N, Binfield PM, King JB (2003) Articular cartilage lesions in the symptomatic anterior cruciate ligament-deficient knee. Arthroscopy 19:685-690

5. McAllister DR, Tsai AM, Dragoo JL, McWilliams J, Dorey FJ, Hame SL, Finerman GAM (2003) Knee function after anterior cruciate ligament injury in elite collegiate athletes. Am J Sports Med 31:560-563
6. Shapiro ET, Richmond JC, Rockett SE, McGrath MM, Donaldson WR (1996) The use of a generic, patient-based health assessment (SF-36) for evaluation of patients with anterior cruciate ligament injuries. Am J Sports Med 24:196-200

7. Mohtadi N (1998) Development and validation of the quality of life outcome measure (questionnaire) for chronic anterior cruciate ligament deficiency. Am J Sports Med 26:350-359

8. Calvisi V, Lupparelli S, De Vincentiis B, Zanoli G (2008) Comorbidity-related quality of life in anterior cruciate ligament insufficiency. A cross-sectional study in 282 candidates to arthroscopic reconstruction. Acta Orthop 79(4):519-525

9. Hacker SA (2003) Chronic anterior cruciate ligament injuries. In: Callaghan JJ, Rosenberg AG, Rubash HE, Simonian PT, Wickiewicz TL (eds) The adult knee. Lippincott Williams \& Wilkins, Philadelphia, pp 717-732

10. Bach BR, Warren RF, Flynn WM, Kroll M, Wickiewiecz TL (1990) Arthrometric evaluation of knees that have a torn anterior cruciate ligament. J Bone Joint Surg Am 72:1299-1306

11. Apolone G, Mosconi P (1998) The Italian SF-36 Health Survey: translation, validation and norming. J Clin Epidemiol 51: $1025-1036$

12. Bryant D, Stratford P, Marx R, Walter S, Guyatt G (2008) Patients can provide a valid assessment of quality of life, functional status, and general health on the day they undergo knee surgery. J Bone Joint Surg Am 90:264-270

13. Ware JE Jr, Snow KK, Kosinski M, Gandek B (1993) The SF-36 health survey manual and interpretation guide. The Health Institute New England Medical Centre, Boston

14. Ware JE Jr, Kosinski M, Bayliss MS, McHorney CA, Rogers WH, Raczek A (1995) Comparison of methods for the scoring and statistical analysis of SF-36 health profile and summary measures: summary of results from the Medical Outcomes Study. Med Care 33(4 Suppl):AS264-AS279

15. Sangha O, Stucki G, Liang MH, Fossel AH, Katz JN (2003) The Self-Administered Comorbidity Questionnaire: a new method to assess comorbidity for clinical and health services research. Arthritis Rheum 49:156-163

16. Hefti F, Muller W, Jakob RP, Staubli HU (1993) Evaluation of knee ligament injuries with the IKDC form. Knee Surg Sports Traumatol Arthrose 1:226-234

17. Ware JE Jr, Bjorner JB, Kosinski M (2000) Practical implications of item response theory and computerized adaptive testing: a brief summary of ongoing studies of widely used headache impact scales. Med Care 38(9 Suppl):II73-82

18. Hjermstad MJ, Fayers PM, Bjordal K, Kaasa S (1998) Using reference data on quality of life. The importance of adjusting for age and gender, exemplified by the EORTC QLQ C-30 (+3). Eur J Cancer 34(9):1381-1389

19. Zanoli G, Jonsson B, Stromqvist B (2006) SF-36 in degenerative lumbar spine disorders. Analysis of prospective data from 451 patients. Acta Orthop 77:298-306

20. McAllister DR, Motamedi AR, Hame SL, Shapiro MS, Dorey FJ (2001) Quality of life assessment in elite collegiate athletes. Am J Sports Med 29:806-810

21. Padua R, Padua S, Aulisa L, Ceccarelli E, Padua L, Romanini E, Zanoli G, Campi A (2001) Patient outcome after Harrington instrumentation for idiopathic scoliosis: a 15 - to 28 -year evaluation. Spine 26(11):1268-1273

22. Padua R, Bondì R, Ceccarelli E, Campi A, Padua L (2008) Health-related quality of life and subjective outcome after shoulder replacement for proximal humeral fractures. J Shoulder Elbow Surg 17(2):261-264

23. Marx RG, Jones EC, Atwan NC, Closkey RF, Salvati EA, Sculco TP (2005) Measuring improvement following total hip and knee arthroplasty using patient-based measures of outcome. J Bone Joint Surg Am 87:1999-2005 
24. Zanoli G (2005) Outcome assessment in lumbar spine surgery. Acta Orthop Suppl 76(318):5-47

25. Jackowski D, Guyatt G (2003) A guide to health measurement. Clin Orthop Relat Res 413:80-89

26. Beaton DE, Schemitsch E (2003) Measures of health-related quality of life and physical function. Clin Orthop Relat Res 413:90-105

27. Apolone G, Mosconi P, Ware JE (1997) Questionario sullo stato di salute SF-36. Guerini e Associati, Milano
28. Harryman DT II, Hettrich CM, Smith KL, Campbell B, Sidles JA, Matsen FA III (2003) A prospective multipractice investigation of patients with full-thickness rotator cuff tears: the importance of comorbidities, practice and other covariables on self-assessed shoulder function and health status. J Bone Joint Surg Am 85-A:690-696

29. European Commission Directorate-General for Education and Culture (2005) Europe: beyond gender. The Magazine. Issue 25. http://europa.eu.int/comm/dgs/education_culture/index_en.html 